\title{
Estrogen replacement is protective to the effect of in vitro hypoxia on female rabbit bladder and pelvic floor contractile response
}

\author{
Amy D. Dobberfuhl ${ }^{1}$ (D), Catherine Schuler ${ }^{2}$, Robert E. Leggett ${ }^{2}$, Elise J.B. De ${ }^{3}$ (D) Robert M. Levin $^{2}$ (i) \\ ${ }^{1}$ Department of Urology, Stanford University School of Medicine, Stanford, CA, ${ }^{2}$ Stratton VA Medical Center, Albany, NY, ${ }^{3}$ Department of Urology, Massachusetts General \\ Hospital, Boston, MA, USA
}

Purpose: To explore the effect of estrogen replacement on pelvic floor and bladder contractile response to electrical field stimulation, following in vitro hypoxia in an animal model of surgical menopause.

Materials and Methods: Twelve female adult rabbits were divided into three groups: control, ovariectomy, and ovariectomy with estradiol replacement. At 4 weeks animals were euthanized. Bladder, coccygeus, and pubococcygeus were isolated. Tissues were equilibrated with oxygenated Tyrodes containing glucose and stimulated with electrical field stimulation. Tissues were then stimulated under hypoxic conditions for 1 hour using nitrogenated Tyrodes without glucose. Tissues were then re-oxygenated for 2 hours and stimulated.

Results: Pelvic floor required 10 times the stimulation duration (power) to achieve maximum contraction at $2 \mathrm{~g}$ baseline tension (10 ms duration) when compared to bladder (1 ms duration). Maximal tension generated was significantly greater for bladder than pelvic floor. Coccygeus and pubococcygeus were significantly less sensitive to the effects of hypoxia and had stable contractile response to field stimulation throughout the hour of hypoxia. Hypoxia resulted in progressive and rapid decline of bladder contractile strength. Following hypoxia, pelvic floor contractile recovery was superior to bladder. Improvement in the contractile response of both bladder and pelvic floor, during the period of post-hypoxia re-oxygenation, was significantly greater in ovariectomy animals treated with estradiol replacement.

Conclusions: Replacement of estradiol at time of ovariectomy reduced oxidative stress on tissue and was protective to the effects of hypoxia on pelvic floor and bladder contractile function.

Keywords: Estrogen replacement therapy; Hypoxia; Models, animal; Pelvic floor; Urinary bladder

This is an Open Access article distributed under the terms of the Creative Commons Attribution Non-Commercial License (http://creativecommons.org/licenses/by-nc/4.0) which permits unrestricted non-commercial use, distribution, and reproduction in any medium, provided the original work is properly cited.

\section{INTRODUCTION}

Weakness of the pelvic floor musculature is well described in stress urinary incontinence and pelvic organ prolapse. Low blood flow, hypoxia and ischemia have been implicated as causes of pelvic floor and bladder dysfunction [1]. Clinicians have long recognized the association between urinary symptoms and other pelvic complaints such as pain

Received: 27 June, 2019 • Accepted: 5 February, 2020

Corresponding Author: Amy D. Dobberfuhl (iD https://orcid.org/0000-0003-3451-3878

Department of Urology, Stanford University School of Medicine, 300 Pasteur Drive, Grant S-287, Stanford, California 94305, USA

TEL: +1-650-725-5746, FAX: +1-650-498-5346, E-mail: adobber@stanford.edu 
and bowel dysfunction [2]. Most recently, a number of lower urinary tract dysfunctions in women have been linked to pelvic organ ischemia and hypoxia mediated by low blood flow during periods of low estrogen [1,3-6]. Likewise, many of these symptoms appear to correlate with estrogen depletion at the time of menopause $[7,8]$.

In the female rabbit after ovariectomy, subsequent low estrogen is associated with poor bladder compliance [4], decreased blood flow to the bladder and uterus [1,4,9], decreased blood vessel density in both bladder muscle and urothelium [1,4,9], thinning of urothelium with urothelial hypoxia [5], and decreased bladder contractility [6]. Consequently, the replacement of estrogen after ovariectomy in rabbits has been shown to restore the in vitro and in vivo tissue mechanics of bladder detrusor muscle [3,6]. Prior animal models of pelvic floor dysfunction include the rabbit, which has been extensively studied and chosen for its well developed pelvic floor [2,10]. The pubococcygeus and coccygeus pelvic floor musculature of the rabbit has been shown to play a central role in mediating bladder storage and micturition as demonstrated by prior investigators (Fig. 1A) [11]. What has not been directly elucidated is the role of estrogen depletion and replacement on the contractile response of the pelvic floor and bladder detrusor muscle in the setting of in vitro hypoxia.

In the present investigation, we sought to characterize the in vitro contractile response of bladder, pubococcygeus and coccygeus muscle strips in the setting of surgically induced menopause with and without estrogen replacement. Biochemical analyses were performed for malondialdehyde, a marker of oxidative stress [12].

\section{MATERIALS AND METHODS}

\section{Animal methods}

All methods were approved by the Stratton VA Institutional Animal Care and Use Committee. Twelve adult female rabbits $(\sim 3 \mathrm{~kg})$ were divided into three groups of four animals each (sham control animal group [control], ovariectomy animal group [OVX], ovariectomy with estradiol replacement animal group [OVX+E]). Each rabbit was anesthetized with isoflurane ( $1 \%$ to $3 \%$ ) through inhalation. In animals which underwent ovariectomy, the ovaries were exposed and excised through a low midline incision and the wound closed in layers with $2-0$ silk. In the sham control group, animals underwent a sham operation where the ovaries were exposed without removing them and then the wound closed. In OVX+E animals, following the completion of ovariectomy, a slow release estrogen tablet was placed subcutaneously in the subscapular region which released $1 \mathrm{mg}$ estradiol per day. All rabbits were then allowed to recover for 4 weeks.

At 4 weeks following surgery, all animals were anesthetized with ketamine/xylazine (25 mg ketamine/10 mg xylazine intramuscular injection per $\mathrm{kg}$ body weight) and then euthanized with pentobarbital in accordance with institutional guidelines. The bladder and entire pelvic floor were rapidly excised and placed in oxygenated cell culture media (\#MT-15-013-CV; Fisher Scientific, Waltham, MA, USA) in preparation for organ bath myography. The bladder body and base were separated and weighed. Three full thickness longitudinal strips of bladder were isolated for physiological testing. Concurrent to the bladder dissection, three coccygeus and three pubococcygeus pelvic floor strips, approximately $150 \mathrm{mg}$ each, were isolated for organ bath myography (Fig. 1A) [11]. The remaining bladder and pelvic floor were then frozen using liquid nitrogen and stored at $-80^{\circ} \mathrm{C}$ to be used as no hypoxia tissue for malondialdehyde analysis.

\section{Physiological analysis}

Each longitudinal strip of bladder, pubococcygeus and coccygeus were mounted in separate $15 \mathrm{~mL}$ organ baths containing oxygenated ( $95 \%$ oxygen and $5 \%$ carbon dioxide) Tyrodes solution at $37^{\circ} \mathrm{C}$. All strips were set at $2 \mathrm{~g}$ preload tension and equilibrated for 30 minutes. Preliminary studies demonstrated that maximum contractile response for muscle strips this size is obtained at $2 \mathrm{~g}$ preload tension. One strip of pubococcygeus and one strip of coccygeus underwent electrical field stimulation to confirm duration response at $32 \mathrm{~Hz}$ (stimulation duration 1, 2, 4, 6, 8, and $10 \mathrm{~ms}$ ). This confirmed our previous studies which demonstrated that maximal stimulation of bladder occurs at $1 \mathrm{~ms}$ stimulation duration. Following this, all bladder strips underwent electrical field stimulation frequency response testing $(2,8$, and $32 \mathrm{~Hz} ; 1 \mathrm{~ms}$ duration for 20 seconds; 3 minute interval between stimulation). Similarly, all pelvic floor strips underwent electrical field stimulation frequency response testing $(2,8$, and $32 \mathrm{~Hz}$; $10 \mathrm{~ms}$ duration for 20 seconds; 3 minute interval between stimulation).

After all pre-hypoxia electrical field stimulation was complete; tissues were exposed to in vitro hypoxia. Organ bath solution was exchanged for Tyrodes without glucose and equilibrated with 95\% nitrogen and 5\% carbon dioxide for 1 hour with stimulation at $32 \mathrm{~Hz}$ occurring every 5 minutes (bladder tissue stimulation duration of $1 \mathrm{~ms}$; pubococcygeus and coccygeus tissue stimulation duration of $10 \mathrm{~ms}$ ).

At the completion of the hypoxic period, all tissues underwent re-oxygenation. The organ bath solution was changed back to oxygenated Tyrodes containing glucose, and tissue strips were allowed to recover for two hours. The 
A

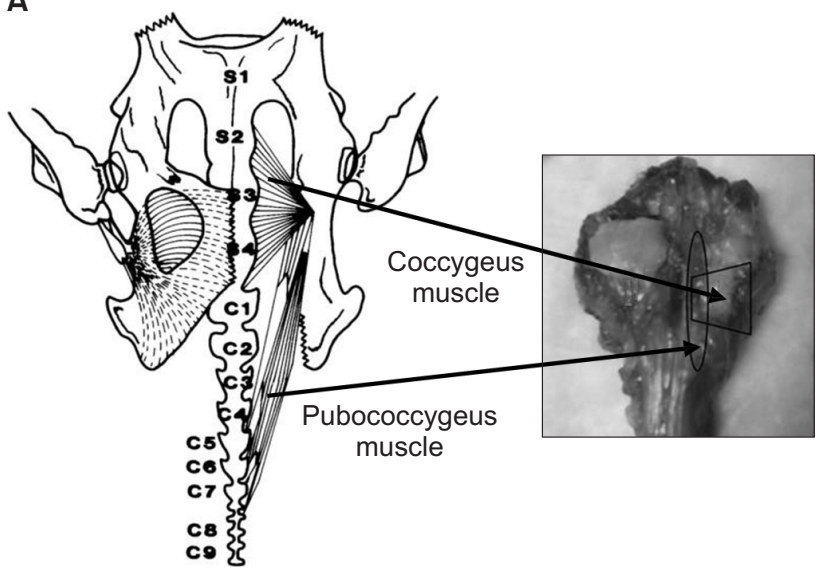

C

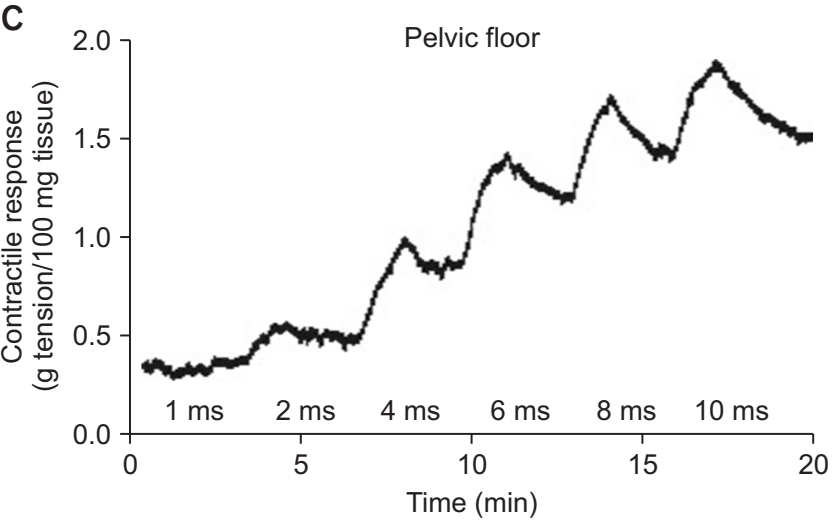

$E$

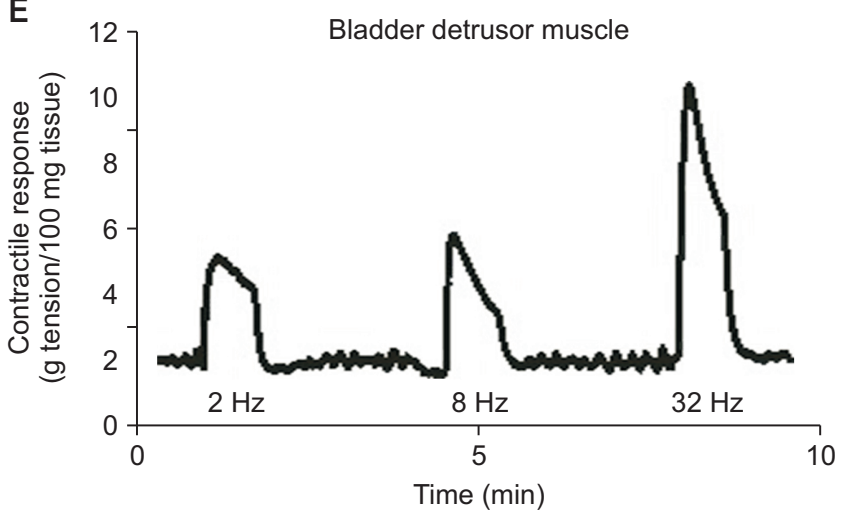

B

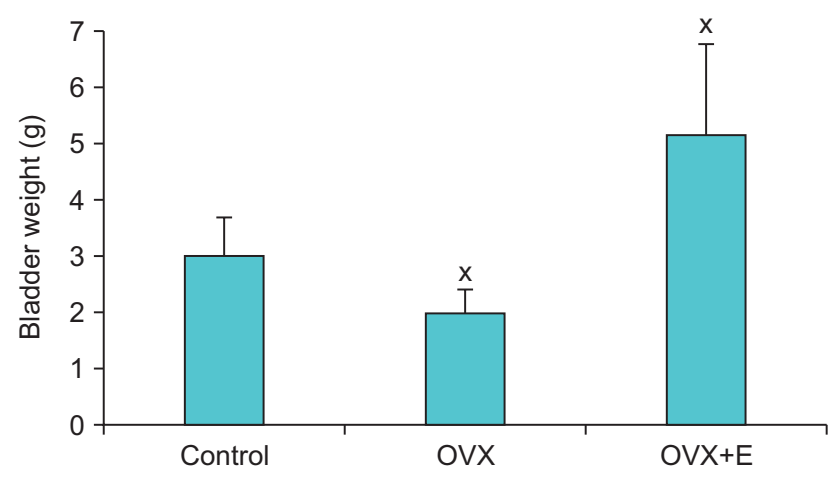

D $2.0 \quad$ Pelvic floor

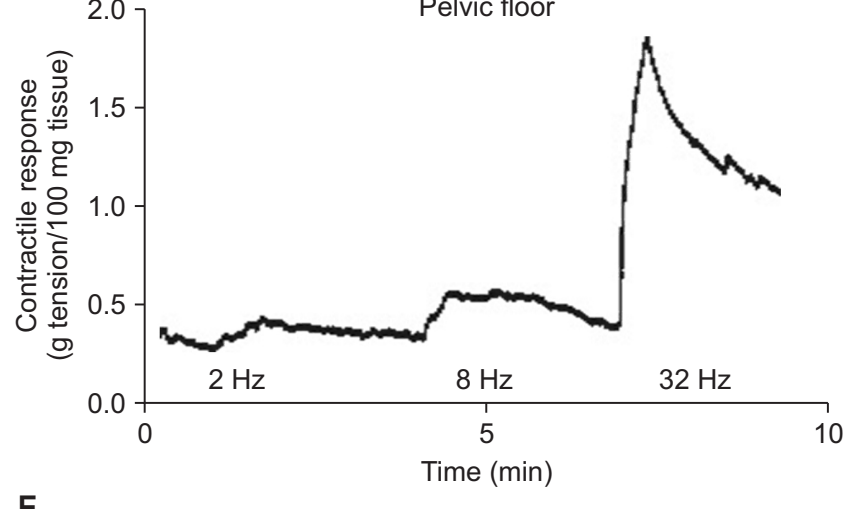

$\mathbf{F}$

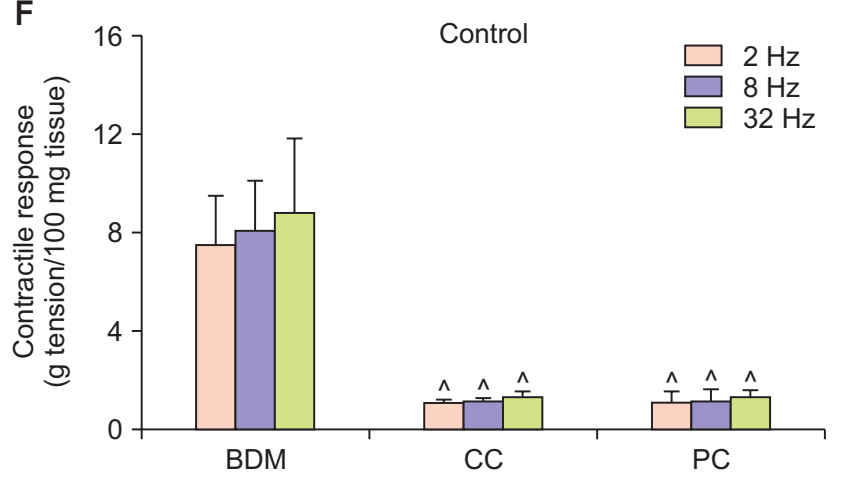

Fig. 1. (A) Diagram of the rabbit pelvic floor musculature adapted from Martínez-Gómez et al. [11], with permission (RightsLink license number 3834880639817). The pubococcygeus muscle pulls the fused sacral vertebrae and tail ventrally. The coccygeus muscle is responsible for ipsilateral abduction of tail, vagina and rectum. (B) Effect of ovariectomy and estrogen replacement on bladder weight. Each bar is the mean \pm standard deviation of 4 individual rabbits; $x=$ significantly different from sham control animal group $(p<0.05)$. Control, sham control animal group; OVX, ovariectomy animal group; $\mathrm{OVX}+\mathrm{E}$, ovariectomy with estradiol replacement animal group. (C) Representative example of the duration response of the pelvic floor to electrical field stimulation $(1$ to $10 \mathrm{~ms}$ duration at $32 \mathrm{~Hz}$ ). (D) Representative example of the frequency response of the pelvic floor to electrical field stimulation $(2,8,32 \mathrm{~Hz}$ at $10 \mathrm{~ms}$ duration). (E) Representative example of the frequency response of the bladder to electrical field stimulation $(2,8,32 \mathrm{~Hz}$ at 1 ms duration). (F) Maximum contractile response of the three muscle types (bladder, coccygeus and pubococcygeus) to electrical field stimulation in sham control animals $(2,8,32 \mathrm{~Hz})$. Each bar is the mean \pm standard deviation; $\wedge=$ significantly different from bladder detrusor muscle $(p<0.05)$. BDM, bladder detrusor muscle; $C C$, coccygeus; $\mathrm{PC}$, pubococcygeus.

strips were then stimulated with electrical field stimulation as previously described to assess change in frequency response [2, 8, and $32 \mathrm{~Hz} ; 1 \mathrm{~ms}$ (bladder) and $10 \mathrm{~ms}$ (pubococcygeus and coccygeus) stimulation duration for 20 seconds; 3 minute interval between stimulation]. The contractile seg- ment of each muscle strip was then cut from the strip holders and weighed for normalization of tension generated and comparison between strips ( $\mathrm{g}$ tension/100 mg tissue). Strips were then frozen using liquid nitrogen for subsequent malondialdehyde analysis. 


\section{Biochemical analyses for malondialdehyde}

Each frozen tissue sample was homogenized using $\mathrm{KCl}$ Tris buffer $(1.15 \% \mathrm{KCl}$ per $0.005 \mathrm{M}$ Tris buffer, $\mathrm{pH}$ 7.4, at 20 $\mathrm{mg} / \mathrm{mL}$ ). Homogenates were spun at $800 \mathrm{~g}$ for 10 minutes, and the supernatants were saved. Ferrous sulfate, $25 \mu \mathrm{L}$, was added to $1.25 \mathrm{~mL}$ of each supernatant and incubated in a water bath at $37^{\circ} \mathrm{C}$ for 30 and 60 minutes. A zero time point (basal value) for each sample was generated. At the end of each incubation period, the reaction was stopped by adding $100 \mu \mathrm{L}$ of each sample to mini centrifuge tubes containing $30 \mu \mathrm{L}$ of $40 \%$ trichloroacetic acid. Samples were then centrifuged at 1,000g for 2 minutes. After centrifugation, 100 $\mu \mathrm{L}$ of each sample was added to $750 \mu \mathrm{L}$ of $1 \%$ thiobarbituric acid solution ( $\mathrm{pH} 7.4$ ), in $1.2 \mathrm{~mL}$ test tubes. Samples were then incubated at $90^{\circ} \mathrm{C}$ for 30 minutes. Samples were then allowed to cool on ice for 10 minutes. Following this, $2 \mathrm{~mL}$ of 1-butanol was added to each tube and spun at 1,000 $\mathrm{g}$ for 5 minutes. The upper layer was removed and measured at 532 nm using a Hitachi F-2500 spectrophotometer (Hitachi HighTechnologies Corporation, Tokyo, Japan). Quantification of total protein was performed using a Micro BCA protein assay kit (Pierce, Rockford, IL, USA). Results are expressed for malondialdehyde in $\mu \mathrm{mol} / \mathrm{mg}$ protein.

\section{Statistical analysis}

Data were analyzed in SigmaPlot (Systat Software Inc., San Jose, CA, USA). Results are expressed as mean \pm standard deviation. Contractile tension was normalized to grams of tension generated per $100 \mathrm{mg}$ tissue for all analyses. Oneway analysis of variance was used for between group comparisons, to determine if significant differences were present between the three animal groups. The Tukey test was used to determine which animal groups were significantly different, and to account for multiple comparisons. The Student's t-test was used for within group comparison during and after hypoxia, referenced to each group's maximal and initial contraction respectively. A p $<0.05$ was required for statistical significance.

\section{RESULTS}

Bladder weight was significantly decreased in the ovariectomy (OVX) treatment group and restored above control values in the estradiol replacement $(\mathrm{OVX}+\mathrm{E})$ animal group (Fig. 1B). Both pelvic floor muscle groups (pubococcygeus and coccygeus) required 10 times the stimulation duration (power) to achieve maximum contraction at $2 \mathrm{~g}$ baseline tension (10 ms stimulation duration) when compared to bladder which was able to achieve maximum contraction at $1 \mathrm{~ms}$ stimula- tion duration. An electrical field stimulation duration of 1 $\mathrm{ms}$ to achieve maximum contractile response for bladder tissue is consistent with previous investigations [13]. A characteristic duration response curve for the pelvic floor musculature at $32 \mathrm{~Hz}$ is shown (Fig. 1C). Similarly, the frequency response curve for pelvic floor at $10 \mathrm{~ms}$ stimulation duration (Fig. 1D), and bladder at $1 \mathrm{~ms}$ stimulation duration (Fig. 1E) are illustrated.

Maximal contractile response was significantly greater for bladder than either pubococcygeus or coccygeus (Fig. 1F). Ovariectomy resulted in significantly decreased contractile responses at all stimulation frequencies in all three tissues tested (Fig. 2). Estradiol replacement (OVX+E) animals demonstrated an increased contractile response compared to sham control animals at all frequencies for both the bladder and coccygeus muscle groups; whereas the OVX+E pubococcygeus muscle only demonstrated an increased contractile response at $32 \mathrm{~Hz}$.

In the setting of hypoxia, there was a progressive decline in bladder contractile strength over time, with near complete loss of response after 1 hour of hypoxia (Fig. 3). Both coccygeus and pubococcygeus were significantly less sensitive to the effects of hypoxia and had stable but reduced contractile response to $32 \mathrm{~Hz}$ of electrical field stimulation throughout the hour of hypoxia. Ovariectomy without estradiol replacement (OVX) had no effect on hypoxia compared to control tissue for either of the pelvic floor muscle groups. Estradiol replacement in OVX+E animals had no effect on the contractile responses of the coccygeus muscles in the setting of hypoxia; whereas estradiol significantly improved the contractile responses of the bladder and pubococcygeus tissues, when compared to ovariectomy without estradiol replacement (OVX) animals.

Following 2 hours of re-oxygenation, bladder tissue contractile response was significantly reduced for the ovariectomy without estradiol replacement (OVX) animals. Estradiol replacement in OVX+E animals was accompanied by a significant increase in contractile response for all tissues during the re-oxygenation phase, when compared to sham control and OVX groups (Fig. 4).

Malondialdehyde, a biomarker for oxidative stress, was significantly increased in both bladder and pelvic floor tissues, for all animals which underwent ovariectomy without estradiol replacement (OVX). Estradiol replacement in OVX+E animals was associated with lower levels of oxidative stress, as demonstrated by reduced malondialdehyde levels in both bladder and pelvic floor tissues after hypoxia, when compared to ovariectomy without estradiol replacement (OVX) animals (Fig. 5). 
A
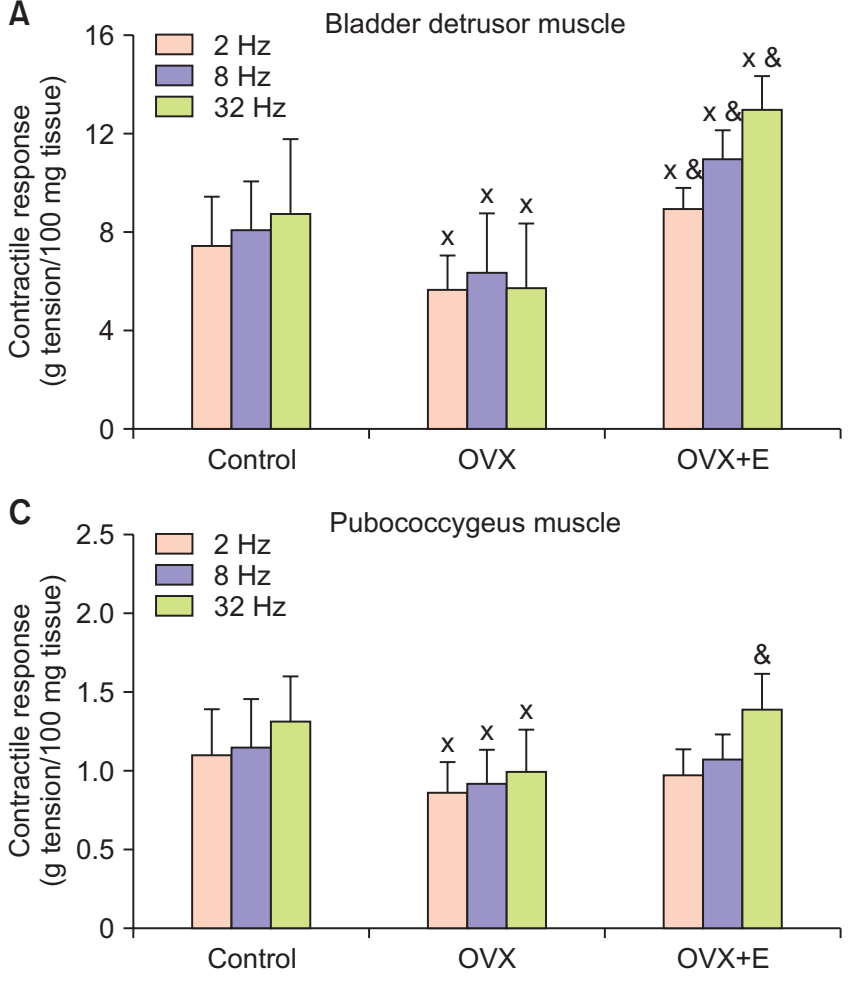

B

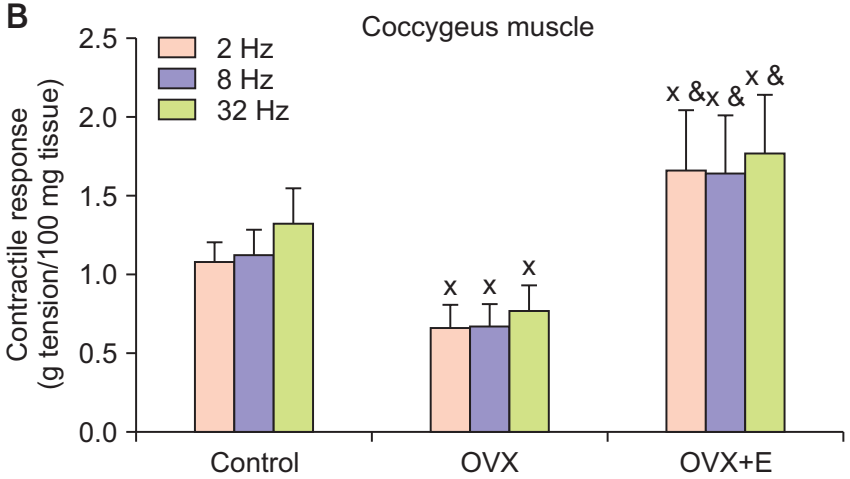

Fig. 2. Effect of ovariectomy and estrogen on the contractile response of the bladder and pelvic floor (coccygeus and pubococcygeus) to electrical field stimulation $(2,8,32 \mathrm{~Hz})$. Tension calculated in grams per $100 \mathrm{mg}$ of tissue. Each bar is the mean \pm standard deviation of 4 individual rabbits; $x=$ significantly different from sham control animal group $(p<0.05)$; $\&=$ significantly different from OVX animal group $(p<0.05)$. Control, sham control animal group; OVX, ovariectomy animal group; $\mathrm{OVX}+\mathrm{E}$, ovariectomy with estradiol replacement animal group.

\section{DISCUSSION}

In rabbits, as in humans, estrogen depletion has been shown to cause genitourinary hypoxia with both pathologic and physiologic consequences, including decreased blood flow to the bladder and uterus, thinning of the bladder urothelium, urothelial hypoxia and poor bladder compliance [4,6,14,15]. Bladder ischemia has been implicated in the manifestation of detrusor overactivity during the acute phase of bladder outlet obstruction, and detrusor underactivity during the chronic decompensated phase of obstruction [16]. Recent investigations have demonstrated a therapeutic role for the phosphodiesterase type5 inhibitor (tadalafil) in the treatment of lower urinary tract dysfunction in animal models of lower urinary tract ischemia [17-19]. Similarly, symptoms attributed to overactive bladder have been shown to correlate with estrogen depletion [7] and response to anticholinergics has been shown to improve with the addition of estrogen therapy [20]. In light of these potential therapies which may exert differential pharmacologic effects, dependent on the hypoxic and estrogenic milieu of the bladder and pelvic floor musculature, we sought to further characterize the effect of estradiol in an animal model of surgical menopause, and further explore the sensitivity of pelvic floor and bladder muscle contractile response following in vitro hypoxia.
In our current study we demonstrated that ovariectomy in the rabbit is associated with a significant decrease in bladder weight, with a restoration above control value after estrogen replacement. Estrogen replacement of $1 \mathrm{mg}$ estradiol per day in the rabbit has been shown to restore estrogen levels to at least physiologic normality based on our prior investigations [3,4]. Following in vitro re-oxygenation we demonstrated that bladder tissue was significantly more sensitive to the effects hypoxia than the pelvic floor. Hypoxia and subsequent re-oxygenation without estradiol replacement in OVX animals resulted in decreased contractile response for all tissues; whereas estradiol replacement in OVX+E animals restored contractile response above and beyond the contractile response demonstrated by sham control animals.

Our experiment presents evidence that both the bladder and pelvic floor muscles are sensitive to estrogen depletion and the effects are almost completely reversed by estrogen replacement. The improvement in contractile function may partially explain the symptomatic improvement in lower urinary tract symptoms that we see in humans after estrogen therapy [7]. Our biochemical analysis of malondialdehyde demonstrates an increase in oxidative stress in the setting of estrogen depletion. This is in concordance with other investigators who have found that estrogen depletion results 

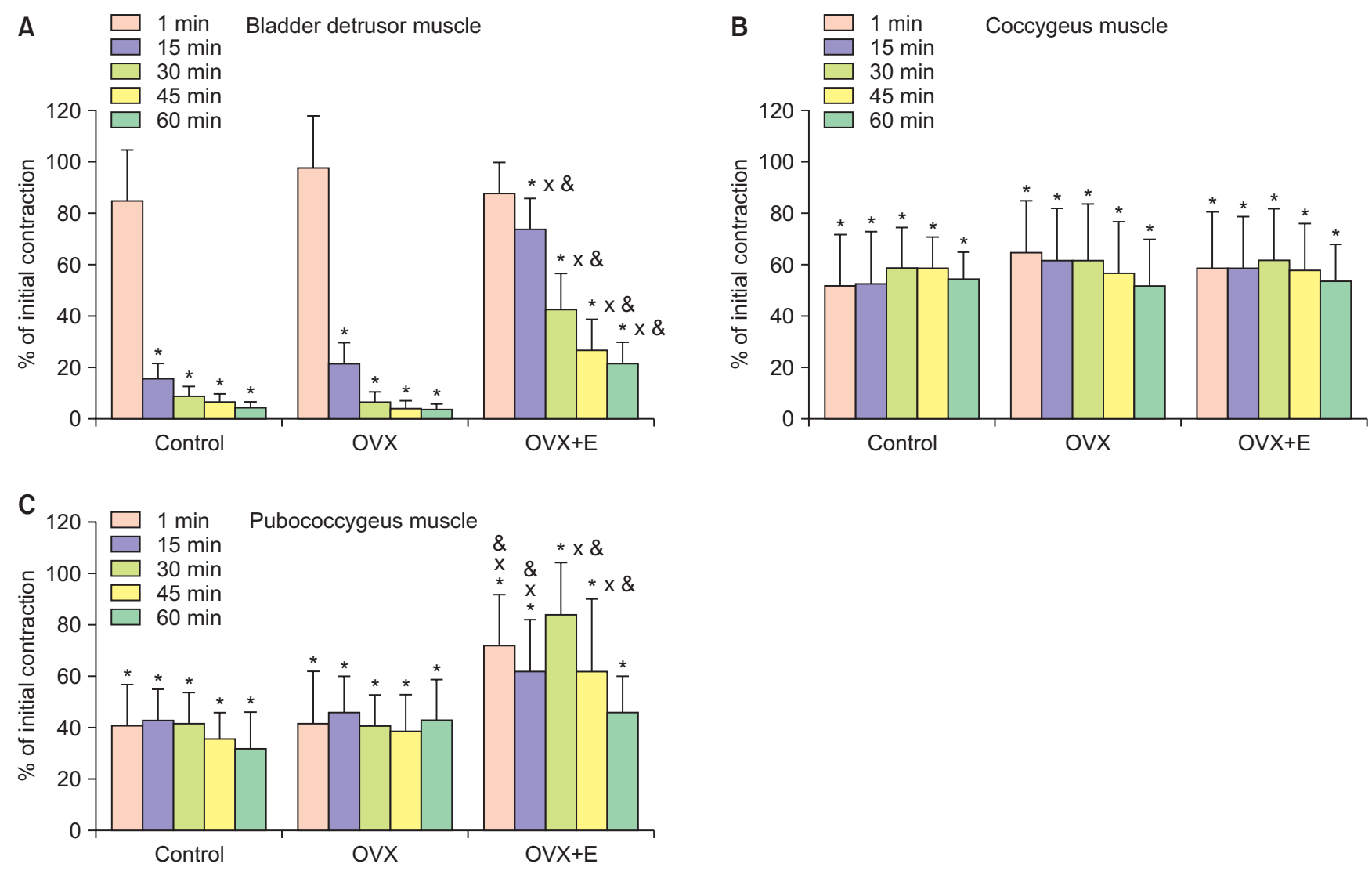

Fig. 3. Effect of ovariectomy and estrogen on the contractile responses of the bladder and pelvic floor (coccygeus and pubococcygeus) to $32 \mathrm{~Hz}$ electrical field stimulation during hypoxia (1, 15, 30, 45, 60 minutes). Tension calculated in grams per 100 mg of tissue, and expressed as percent of maximum initial contraction obtained at $32 \mathrm{~Hz}$ for each tissue strip prior to hypoxia. Each bar is the mean \pm standard deviation of 4 individual

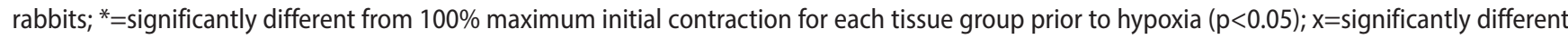
from sham control animal group ( $p<0.05) ; \&=$ significantly different from OVX animal group $(p<0.05)$. Control, sham control animal group; OVX, ovariectomy animal group; OVX+E, ovariectomy with estradiol replacement animal group.

in a significant increase in oxidative stress, and as expected estrogen replacement significantly attenuates oxidative stress $[1,4,8,9,21]$.

Pelvic floor ischemia is poorly characterized in the literature and the exact association with bladder dysfunction is not completely understood. Transient hypoxia of the pelvic floor has been implicated as an important factor during child birth injury [22]. Pelvic floor hypoxia is also thought to be related to pelvic pain and hypothesized to play a role in pelvic floor spasm. Vaginal distension models have been shown to result in pelvic floor ischemia [23]. The pelvic floor consists primarily of slow twitch skeletal muscles with a high resting tone important for the support of pelvic organs and the maintenance of fecal and urinary continence. In the setting of pelvic floor dysfunction, either a hyper or hypotonic pelvic floor can manifest as lower urinary tract symptoms with associated chronic pelvic pain, dyspareunia and voiding dysfunction. Further research is needed to explore the effect of estrogen on the pelvic floor in humans.

In our current study, we are the first to present data on the electrical field stimulation response of the rabbit pelvic floor musculature in the setting of hypoxia and estrogen replacement. The coccygeus and pubococcygeus muscle groups have been well characterized in the rabbit pelvic floor literature (Fig. 1A), with the pubococcygeus functioning as a muscle which causes ipsilateral abduction of the tail, vagina and rectum. Meanwhile the coccygeus pulls the fused sacral vertebrae and tail ventrally [11]. As the most homologous counterparts to the human pelvic floor, these muscle groups have been shown to play an important role in voiding and defecatory function in the quadruped rabbit. Our results demonstrated that the pelvic floor musculature were slower to contract when compared to bladder detrusor muscle, and the pelvic floor required 10 times the electrical field stimulation duration (power) to achieve maximum contraction. This is consistent with the cyclic function of the detrusor muscle, which during a voiding contraction undergoes a large change in volumetric size in a relatively short time frame; meanwhile the pelvic floor contraction length is minimally changed during relaxation at the time of void. 
A

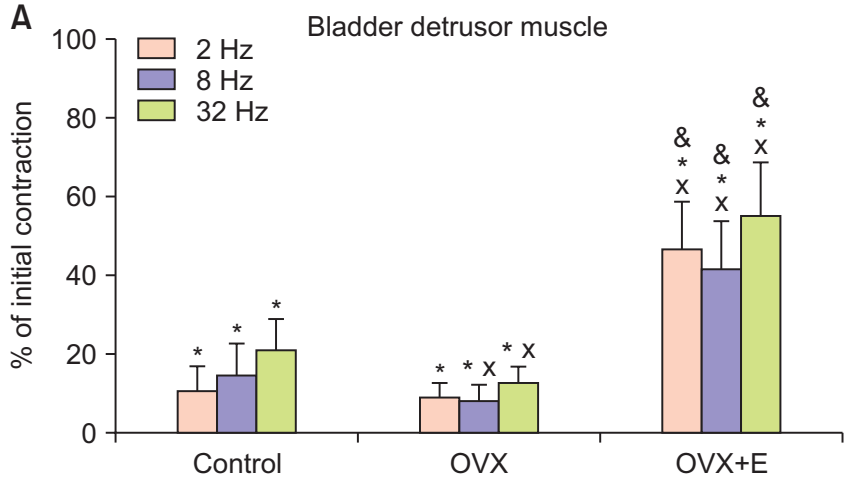

C

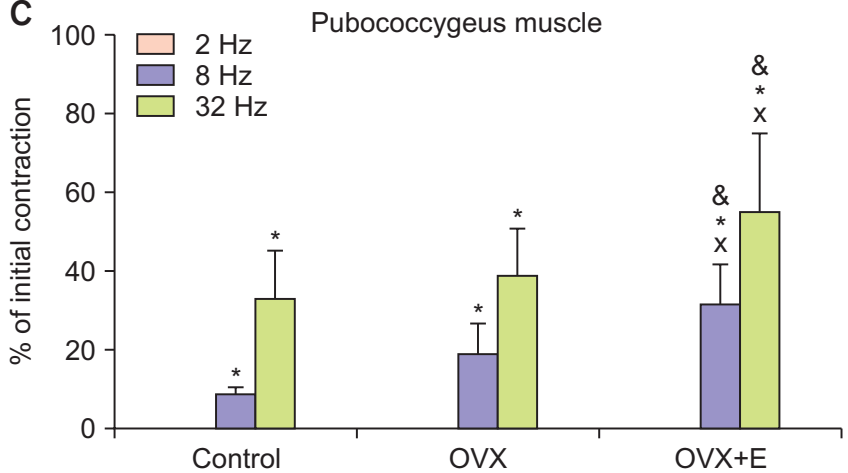

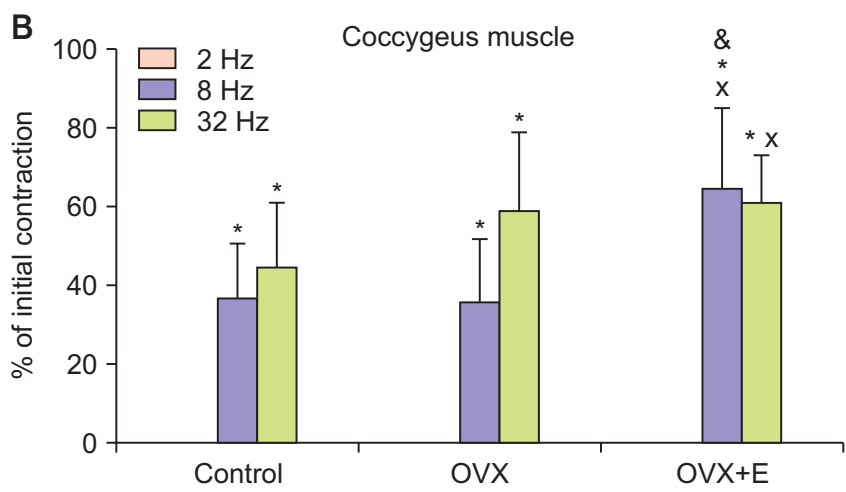

Fig. 4. Effect of ovariectomy and estrogen on the contractile responses of the bladder and pelvic floor (coccygeus and pubococcygeus) to electrical field stimulation following 2 hours of re-oxygenation $(2,8,32 \mathrm{~Hz})$. Tension calculated in grams per $100 \mathrm{mg}$ of tissue, and expressed as percent of maximum initial contraction obtained at 2, 8, and $32 \mathrm{~Hz}$ for each strip prior to hypoxia. Each bar is the mean \pm standard deviation of 4 individual

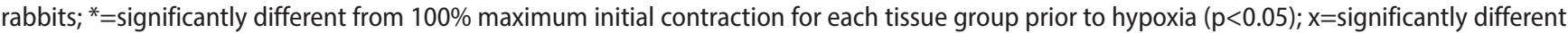
from sham control animal group ( $p<0.05)$; \&=significantly different from OVX animal group $(p<0.05)$. Control, sham control animal group; OVX, ovariectomy animal group; $\mathrm{OVX}+\mathrm{E}$, ovariectomy with estradiol replacement animal group.

In the setting of hypoxia, all of the pelvic floor tissues demonstrated a reduced but consistent contractile response to field stimulation during the entire hour of hypoxia. The pubococcygeus muscle from the OVX+E animal group demonstrated improvement in contractile response during the ischemic period; whereas coccygeus muscle contractile response was the same throughout hypoxia, regardless of the level of estrogen. Limitations of our study include the following. We chose the rabbit to model the pelvic floor, however as previously stated, as a quadruped the rabbit pelvic floor does not support the pelvic organs in the same manner as the upright human. Due to the large size of the animal and animal care and use regulations limiting animal use to the lowest number to achieve meaningful results, we were limited to four animals per treatment group. The rabbit however has been shown to utilize the pelvic floor in a similar manner to humans for voiding and defecatory function, as such we believe our findings may be generalizable [2,10]. This is one of the first experiments to characterize the differential effect of the bladder and pelvic floor musculature contractile responses to estrogen depletion and replacement in the setting of hypoxia.
Our findings are consistent with prior investigators, thus reinforcing the physiologic role of estrogen therapy in the treatment of lower urinary tract dysfunction. Our study agrees with the growing body of evidence emphasizing the important role of the pelvic floor in modulating voiding function and provides some insight into the relative contractile responses of the pelvic floor and detrusor muscle in the setting of hypoxia followed by re-oxygenation.

\section{CONCLUSIONS}

We found that replacement of estrogen following ovariectomy reduced oxidative stress on the bladder and pelvic floor, and was protective to the effects of hypoxia on contractile function. Future clinical studies are warranted to explore the therapeutic effect of estrogen on the pelvic floor musculature in humans with suspected ischemic pelvic floor dysfunction.

\section{CONFLICTS OF INTEREST}

The authors have nothing to disclose. 
A

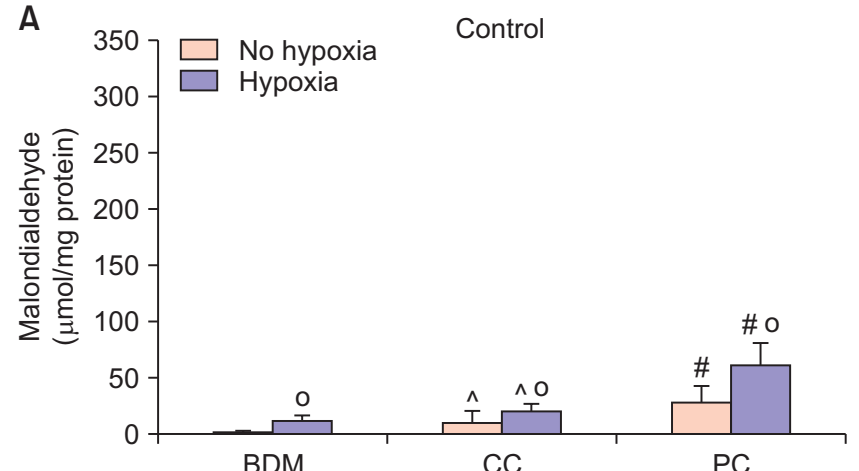

C

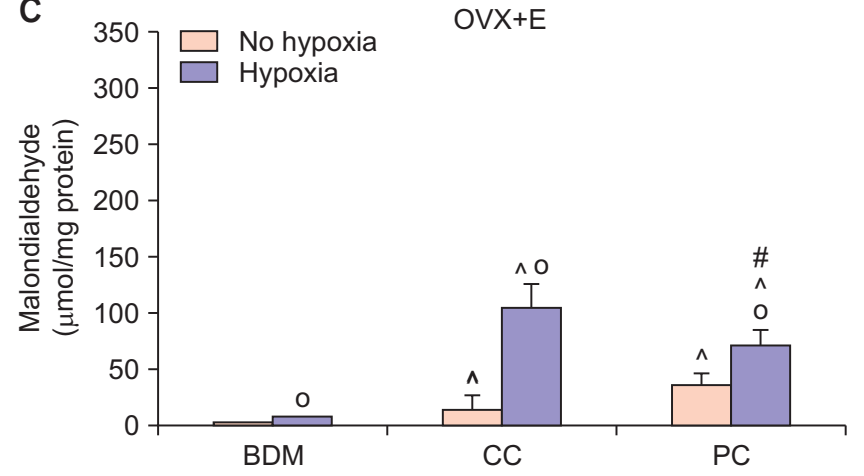

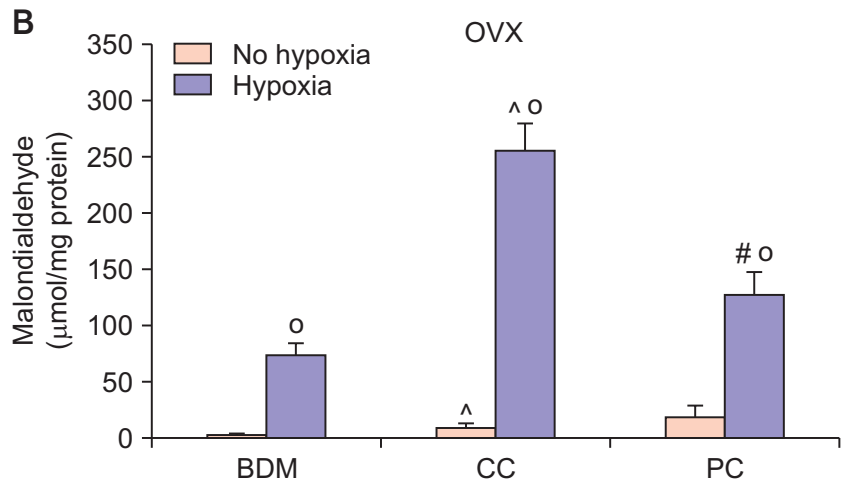

Fig. 5. Effect of ovariectomy and estrogen on the malondialdehyde content ( $\mu \mathrm{mol} / \mathrm{mg}$ protein) of the bladder and pelvic floor (coccygeus and pubococcygeus) following hypoxia. Hypoxia tissue was obtained at end of experiment following 2 hours of re-oxygenation. Tissue without exposure to hypoxia (no hypoxia group) was obtained at start of experiment, and did not undergo organ bath testing. Each bar is the mean \pm standard deviation of 4 individual rabbits; $0=$ significantly different from No hypoxia group $(p<0.05) ; \wedge=$ significantly different from bladder detrusor muscle ( $p<0.05)$; \#=significantly different from coccygeus muscle ( $p<0.05)$. BDM, bladder detrusor muscle; $C C$, coccygeus; PC, pubococcygeus; Control, sham control animal group; OVX, ovariectomy animal group; $\mathrm{OVX}+\mathrm{E}$, ovariectomy with estradiol replacement animal group.

\section{ACKNOWLEDGMENTS}

This material is based upon work supported in part by the Capital Region Medical Research Foundation and the Office of Research \& Development Department of the Veterans Affairs.

The project described herein was conducted with author (ADD) support from the KL2 component of the Stanford Clinical Translational Science Award to Spectrum (NIH 5KL2TR001083-05) and NIH Loan Repayment Program (NIH 1L30DK115056-01).

\section{AUTHORS' CONTRIBUTIONS}

Research conception and design: Amy D. Dobberfuhl and Robert M. Levin. Data acquisition: Amy D. Dobberfuhl, Catherine Schuler, Robert E. Leggett, and Robert M. Levin. Statistical analysis: Robert M. Levin. Data analysis and interpretation: Amy D. Dobberfuhl, Catherine Schuler, Robert E. Leggett, Elise J.B. De, and Robert M. Levin. Drafting of the manuscript: Amy D. Dobberfuhl, Catherine Schuler, Robert E. Leggett, Elise J.B. De, and Robert M. Levin. Critical revision of the manuscript: Amy D. Dobberfuhl, Elise J.B. De, and Robert M. Levin. Obtaining funding: Robert M. Levin. Administrative, technical, or material support: Catherine Schuler and Robert E. Leggett. Supervision: Amy D. Dobberfuhl, Elise J.B. De, and Robert M. Levin. Approval of the final manuscript: Amy D. Dobberfuhl and Robert M. Levin.

\section{REFERENCES}

1. Palmieri K, Mannikarottu AS, Chichester P, Kogan B, Leggett RE, Whitbeck C, et al. The effects of cyclical oestrogen on bladder and urethral structure and function. BJU Int 2007;99:1716.

2. Dobberfuhl AD, Spettel S, Schuler C, Levin RM, Dubin AH, De EJ. Noxious electrical stimulation of the pelvic floor and vagina induces transient voiding dysfunction in a rabbit survival model of pelvic floor dystonia. Korean J Urol 2015;56:837-44.

3. Juan YS, Mannikarottu A, Kogan BA, Leggett RE, Whitbeck C, Chichester $\mathrm{P}$, et al. The effect of low-dose estrogen therapy on ovariectomized female rabbit bladder. Urology 2008;71:120913.

4. Rehfuss A, Schuler C, Maxemous C, Leggett RE, Levin RM. 
Cyclical estrogen and free radical damage to the rabbit urinary bladder. Int Urogynecol J 2010;21:489-94.

5. Badger WJ, Whitbeck C, Kogan B, Chichester P, Levin RM. The immediate effect of castration on female rabbit bladder blood flow and tissue oxygenation. Urol Int 2006;76:264-8.

6. Parekh MH, Chichester P, Lobel RW, Aikawa K, Levin RM. Effects of castration on female rabbit bladder physiology and morphology. Urology 2004;64:1048-51.

7. Robinson D, Cardozo L, Milsom I, Pons ME, Kirby M, Koelbl $\mathrm{H}$, et al. Oestrogens and overactive bladder. Neurourol Urodyn 2014;33:1086-91.

8. Sánchez-Rodríguez MA, Zacarías-Flores M, Arronte-Rosales A, Correa-Muñoz E, Mendoza-Núñez VM. Menopause as risk factor for oxidative stress. Menopause 2012;19:361-7.

9. Chuang SM, Long CY, Lin RJ, Liu KM, Levin RM, Chang CY, et al. Protective effects of estrogen on ischemia/reperfusioninduced bladder dysfunction in female rabbits. Menopause 2013;20:209-17.

10. Dobberfuhl AD, Spettel S, Schuler C, Dubin AH, Levin RM, De EJ. A novel cystometric model of pelvic floor dysfunction after rabbit pelvic floor noxious electrical stimulation. Female Pelvic Med Reconstr Surg 2016;22:248-53.

11. Martínez-Gómez M, Lucio RA, Carro M, Pacheco P, Hudson R. Striated muscles and scent glands associated with the vaginal tract of the rabbit. Anat Rec 1997;247:486-95.

12. Radu F, Leggett RE, Schuler C, Levin RM. The effect of in vitro ischemia/reperfusion on contraction, free fatty acid content, phospholipid content, and malondialdehyde levels of the rabbit urinary bladder. Mol Cell Biochem 2011;346:179-86.

13. Levin RM, English M, Barretto M, Dubuc M, O'Connor L, Leggett $\mathrm{R}$, et al. Normal detrusor is more sensitive than hypertrophied detrusor to in vitro ischemia followed by re-oxygenation. Neurourol Urodyn 2000;19:701-12.

14. Huang FH, Zhang XL, Xiao LP, Xu YY, Zhang YZ. [Study of blood flow improvement of topical application of estrogen on low genitourinary tract and its safety]. Zhonghua Yi Xue Za Zhi 2012;92:2778-80. Chinese.

15. Long CY, Liu CM, Hsu SC, Chen YH, Wu CH, Tsai EM. A randomized comparative study of the effects of oral and topical estrogen therapy on the lower urinary tract of hysterectomized postmenopausal women. Fertil Steril 2006;85:155-60.

16. Andersson KE, Nomiya M, Yamaguchi O. Chronic pelvic ischemia: contribution to the pathogenesis of lower urinary tract symptoms (LUTS): a new target for pharmacological treatment? Low Urin Tract Symptoms 2015;7:1-8.

17. Andersson KE, de Groat WC, McVary KT, Lue TF, Maggi M, Roehrborn CG, et al. Tadalafil for the treatment of lower urinary tract symptoms secondary to benign prostatic hyperplasia: pathophysiology and mechanism(s) of action. Neurourol Urodyn 2011;30:292-301.

18. Kawai Y, Oka M, Yoshinaga R, Fuchikami C, Oyama T. Effects of the phosphodiesterase 5 inhibitor Tadalafil on bladder function in a rat model of partial bladder outlet obstruction. Neurourol Urodyn 2016;35:444-9.

19. Maciejewski CC, Tredget EE, Metcalfe PD. Urodynamic improvements following oral medical therapy for partial bladder outlet obstruction in an animal model. Neurourol Urodyn 2015;34:286-91.

20. Tseng LH, Wang AC, Chang YL, Soong YK, Lloyd LK, Ko YJ. Randomized comparison of tolterodine with vaginal estrogen cream versus tolterodine alone for the treatment of postmenopausal women with overactive bladder syndrome. Neurourol Urodyn 2009;28:47-51.

21. Rangel-Zuñiga OA, Cruz-Teno C, Haro C, Quintana-Navarro GM, Camara-Martos F, Perez-Martinez P, et al. Differential menopause- versus aging-induced changes in oxidative stress and circadian rhythm gene markers. Mech Ageing Dev 2017;164:41-8.

22. Griffin KM, O'Herlihy C, O'Connell PR, Jones JF. Combined ischemic and neuropathic insult to the anal canal in an animal model of obstetric-related trauma. Dis Colon Rectum 2012;55:32-41.

23. Damaser MS, Whitbeck C, Chichester P, Levin RM. Effect of vaginal distension on blood flow and hypoxia of urogenital organs of the female rat. J Appl Physiol (1985) 2005;98:1884-90. 